\title{
Un multilinguisme en construction : l'éveil de la conscience métalinguistique
}

Louise Dabène et Christèle Ingelmann

\section{(2) OpenEdition \\ 12 Journals}

Édition électronique

URL : https://journals.openedition.org/aile/4914

DOI : 10.4000 /aile.4914

ISSN : 1778-7432

\section{Éditeur}

Association Encrages

\section{Édition imprimée}

Date de publication : 1 septembre 1996

Pagination : 123-138

ISSN : 1243-969X

\section{Référence électronique}

Louise Dabène et Christèle Ingelmann, « Un multilinguisme en construction : l'éveil de la conscience métalinguistique », Acquisition et interaction en langue étrangère [En ligne], 7 | 1996, mis en ligne le 27 juin 2012, consulté le 15 avril 2022. URL : http://journals.openedition.org/aile/4914 ; DOI : https:// doi.org/10.4000/aile.4914

Ce document a été généré automatiquement le 15 avril 2022.

(c) Tous droits réservés 


\title{
Un multilinguisme en construction : l'éveil de la conscience métalinguistique
}

\author{
Louise Dabène et Christèle Ingelmann
}

1 Il relève de l'évidence de dire que les Institutions scolaires se trouvent de plus en plus souvent confrontées à la nécessité de gérer des situations de plurilinguisme. Mais sous cette simple affirmation se cachent, en fait, des situations redoutablement complexes et des problématiques fortement diversifiées : soit que la réalité d'un environnement social - et par conséquent d'un public pédagogique - caractérisé par le multilinguisme impose de faire une place à plusieurs langues à l'intérieur de l'univers éducatif autrement qu'en les considérant comme des disciplines scolaires et objets d'apprentissage en tant que telles, soit qu'une politique volontariste conduise à la mise en œuvre de scolarisations caractérisées par l'usage de plus d'une langue comme objet ou comme médium d'enseignement. À l'intérieur de chacun de ces deux types de situations, on peut évidemment distinguer de nouvelles subdivisions: selon les organisations curriculaires, les fonctions confiées à chacune des langues en présence et les activités pédagogiques qui y sont afférentes, la configuration sociolinguistique, le poids de l'environnement, et plus généralement, l'insertion des espaces de plurilinguisme dans le tissu éducatif, et le degré d'investissement des institutions dans ce type d'opération.

2 L'ensemble de ces situations d'appropriation d'une langue vivante, dont le statut peut donc varier considérablement (L. Dabène, 1994), offre un vaste terrain à l'observation. En particulier, on peut se demander si les processus d'acquisition et/ou d'apprentissage mis en œuvre dans ces contextes fortement diversifiés seront ou non similaires. Ce travail de comparaison peut s'effectuer à travers un phénomène caractéristique, qui accompagne toute appropriation, tout au moins celle qui est menée en milieu scolaire, à savoir la constitution progressive d'une conscience métalinguistique, celle-ci étant entendue, au sens large, comme « une attitude réflexive sur les objets langagiers et leur manipulations » (Gombert, 1990). Dans la perspective didactique qui est la nôtre, c'est 
en effet l'activité métalinguistique du sujet qui retient notre attention, tout au moins telle qu'elle nous apparaît à travers les verbalisations et les discours successifs et même si ceux-ci ne font pas usage d'un métalangage spécialisé.

3 Dans le cas qui nous intéresse, et sur lequel nous allons fonder les quelques considérations qui vont suivre, les composantes de la situation peuvent s'analyser, en gros, comme suit.

4 Il s'agit de travailler, dans le contexte de l'enseignement élémentaire français, à l'intersection de deux axes de réflexion et d'action:

- d'une part celui suscité par la présence, dans les classes de l'enseignement élémentaire, d'élèves en partie allophones, et ayant développé des formes de bilinguisme originales, ce qui s'apparente au premier des cas envisagés ci-dessus, étant entendu que, dans la plupart des cas, on a affaire à des groupes linguistiquement peu homogènes.

- la décision, politique, de la mise en œuvre progressive d'un enseignement d'initiation aux langues étrangères à l'école primaire (EILE), ce qui relèverait du second des cas distingués cidessus.

5 Ces deux axes semblent relever de problématiques assez éloignées, mais des zones de convergence se dessinent cependant: c'est, tout d'abord, le fait que les langues dites "d'origine", en tant que discipline scolaire, tendent à perdre les traits qui les caractérisaient, à savoir :

- public ciblé, limité aux élèves enfants de migrants,

- fort investissement identitaire : la langue d'origine, quel qu'en soit le degré de maîtrise par le sujet, est sentie par celui-ci comme marque identitaire (L. Dabène, 1994),

- relative marginalisation dans la structure éducative.

6 Elles s'apparentent donc progressivement aux autres langues étrangères enseignées dans le cadre de l'EILE, évolution déjà observable dans le cas de l'espagnol et de l'italien.

7 C'est, ensuite, le fait que dans le cas de l'EILE, l'accent est mis sur les compétences communicatives et sur la nécessité de déterminer des objectifs et des stratégies spécifiques, qui peuvent s'écarter relativement des pratiques en usage dans les autres ordres d'enseignement.

Il n'en reste pas moins que cette double problématique risque de confronter l'enfant qui s'y trouve exposé à deux approches didactiques sensiblement différentes : dans un cas on se propose de rendre un sujet partiellement bilingue conscient tout à la fois de ses capacités communicatives et des différents systèmes linguistiques auxquelles cellesci renvoient, et dans l'autre, de construire les linéaments d'une future et hypothétique compétence bilingue à partir d'un état initial de monolinguisme.

9 Un des objectifs prioritaires que l'on peut se proposer, dans de telles conditions, est donc de rechercher un lieu commun, relativement unificateur, où puisse se construire une certaine compétence métalinguistique transversale par rapport à ces différents apprentissages. Cette nécessité nous parait d'autant plus forte qu'il n'existe, dans le système scolaire français - et c'est le cas pour beaucoup d'autres systèmes - aucune éducation générale aux faits de langage, celle-ci étant parcellisée et distribuée aux hasards des apprentissages de différentes langues.

10 C'est dans cette perspective qu'ont été élaborées et expérimentées à Grenoble depuis plusieurs années, inspirées par l'apport anglo-saxon (Language Awareness), des 
propositions pédagogiques que nous avons regroupées sous le terme général d' «Eveil au langage » et dont nous contenterons de rappeler brièvement ici les idées forces.

Il s'agit de susciter, chez l'enfant, à partir d'observations et de manipulations effectuées sur une langue d'appui (L1), que celle-ci soit le français ou éventuellement une langue d'origine, mais aussi sur un ensemble de langues aussi varié que possible, la prise de conscience de ce qu'est l'univers du langage dans sa variété, son fonctionnement et son acquisition. On fait l'hypothèse que ce type de travail est de nature à favoriser tout à la fois la maîtrise raisonnée de la L1 et l'apprentissage des langues étrangères, tout en intégrant l'apport des langues d'origines des enfants alloglottes qu'il contribue ainsi à légitimer. Ce type d'activités constitue donc un poste d'observation particulièrement riche pour le repérage de l'activité métalinguistique des sujets, ce que nous nous proposons de mettre en évidence, après avoir présenté les principales caractéristiques de notre public, par l'analyse de trois séquences portant respectivement sur la temporalité, la notion d'accent, et l'ordre des mots.

Nous avons choisi l'école primaire comme terrain d'expérimentation, et plus particulièrement deux classes de cours moyen première et deuxième année, composées respectivement de vingt-six et vingt-cinq enfants âgés de neuf à onze ans. Il a été observé (Balboni, 1993) que, en règle générale, ce public présente une curiosité spontanée pour les langues; de plus, à cet âge-là, les capacités cognitives des enfants leur permettent de procéder à des opérations mentales relativement complexes dans le cadre d'une analyse métalinguistique, telles que le repérage d'éléments communs ou la substitution (cf. exemple sur l'aztèque). La réflexion métalinguistique suppose en outre que le sujet soit capable de distinguer le signifiant du signifié, ce qui n'est pas le cas des enfants plus jeunes (cf. notamment Pratt et Grieve, 1984).

Dans les classes où nous avons travaillé, certains enfants sont monolingues, mais la majorité ne l'est pas; il s'agit principalement de cas de bilingualité d'enfance, à caractère dominant (Hamers et Blanc, 1983), la langue dominante étant ici très clairement le français - la langue de scolarisation. Il s'agit d'une bilingualité précoce, soit simultanée, soit consécutive, le français étant souvent L1, parfois L2 comme pour cette élève née au Portugal, qui est venue en France vers l'âge de trois ans.

\section{La temporalité}

Dans le cadre d'une séance de réflexion métalinguistique sur la L1 majoritaire d'un de nos groupes - en l'occurrence le français -, nous avons tenté d'amener les enfants à prendre conscience des diverses manifestations langagières à l'œuvre dans l'expression de la temporalité. Un de nos objectifs était de les faire réfléchir sur les différentes valeurs que prennent parfois certains temps de conjugaison. Ainsi, à partir d'exemples phrastiques courts, produits par les enfants eux-mêmes, et grâce à un guidage par questionnement - dans une approche de type inductif -, le groupe a progressivement pris conscience, par exemple, que le " présent simple » avait parfois valeur de futur. En d'autres termes, la réflexion a porté sur la différence entre les concepts exprimés en anglais par time et tense (nous sommes obligés d'avoir recours à l'anglais, le terme français «temps" prêtant malheureusement à confusion). La phrase qui a servi de support à la réflexion, produite par un élève, était la suivante :

- demain, je me déguise en clown 
Elle est apparue au milieu d'autres productions, lors d'une réflexion sur la morphologie verbale et l'adéquation indicateur de temps / temps de conjugaison dans la phrase (avec des exemples tels que ce soir je mangerai des frites). C'est en analysant le temps (time) exprimé par l'indicateur de temps d'une part, et par le verbe d'autre part, qu'un des enfants a remarqué une « anomalie » dans cette phrase.

\begin{tabular}{|c|c|}
\hline \multicolumn{2}{|c|}{ ( $\mathrm{E}$ = élève, $\mathrm{I}$ = institutrice, $\mathrm{C}=$ enquêtrice) : } \\
\hline \multicolumn{2}{|c|}{ [l'élève lit la phrase au tableau] } \\
\hline $\begin{array}{ll}\text { 70. } & \text { E. } \\
1: & \end{array}$ & demain je me déguise \\
\hline 71. I. : & qu'est-ce que tu en penses / comment \\
\hline $\begin{array}{l}\text { 72. E. } \\
\text { 1: }\end{array}$ & je donne l'indicateur \\
\hline 73. I. : & l'indicateur si tu veux \\
\hline $\begin{array}{ll}\text { 74. } E . \\
1:\end{array}$ & demain \\
\hline 75. I.: & demain c'est quand \\
\hline $\begin{array}{ll}\text { 76. } E . \\
\text { 1: }\end{array}$ & c'est le futur \\
\hline $\begin{array}{l}\text { 77. } E \text {. } \\
2:\end{array}$ & c'est plus tard \\
\hline 78. I.: & c'est plus tard \\
\hline $\begin{array}{l}\text { 79. E. } \\
\text { 1: }\end{array}$ & le verbe c'est je me déguise \\
\hline 80. I.: & le verbe c'est je me déguise oui / et alors \\
\hline \multicolumn{2}{|c|}{ [silence de 11 secondes] } \\
\hline $\begin{array}{ll}\text { 81. } & \text { E. } \\
3: & \end{array}$ & $\begin{array}{l}\text { ah demain c'était le futur et il a mis le verbe au présent / demain je me déguiserai [brouhaha] c'est } \\
\text { demain je me déguiserai }\end{array}$ \\
\hline
\end{tabular}

La remarque de l'élève 3 (en 81 ), que nous avons interprétée comme une trace d'émergence de la conscience métalinguistique, a lancé une vive discussion au sein du groupe, sur l'acceptabilité de ce type d'énoncé, générant une réflexion intense. Le premier réflexe de certains enfants a été de juger incorrecte la phrase en question, et d'en proposer une version "correcte» (demain je me déguiserai, cf.81), fondant leurs 
arguments sur la logique observée dans les productions où indicateur de temps et temps de conjugaison correspondent. D'autres enfants l'ont intuitivement jugée acceptable (ben je me déguise ça va hein), sans toutefois pouvoir justifier ce jugement. Le débat qui a opposé les deux groupes a dynamisé la réflexion métalinguistique, dans la mesure où chacun désirait ardemment convaincre son voisin, étant ainsi contraint à justifier son opinion, et par là même à réfléchir et verbaliser sa réflexion. Les élèves ont ainsi abouti aux remarques suivantes, qui soulignent qu'ils ont, pour certains, perçu deux concepts distincts (198), ainsi que le poids de l'indicateur de temps dans l'expression du temps time (127 et 312) :

\begin{tabular}{|l|l|}
\hline 198. E.2 : & et dans une phrase y peut y avoir deux temps différents \\
\hline & \\
\hline 127. E.1: & non parce que c'est grâce à l'indicateur \\
\hline & \\
\hline 311. I.: & c'est pas encore passé / comment on sait que le moment il est futur \\
\hline 312. E.4: & parce que ce soir c'est l'indicateur / l'indicateur il nous dit quand ça va se passer \\
\hline 313. & oui / et la forme du verbe le temps du verbe il est à quel temps le verbe \\
\hline 314. E.2: & au présent il est au présent \\
\hline 315. & oui / et alors on comprend ou on comprend pas \\
\hline 316. E.5: & on comprend \\
\hline
\end{tabular}

Le métalangage utilisé n'est bien évidemment pas très précis, les élèves tentant de définir les concepts en leur propres termes. On peut toutefois percevoir ici une activité métalinguistique, qui n'a peut-être pas complètement abouti à une image claire des concepts time et tense chez tous les enfants; il est néanmoins évident que certains ont touché du doigt cette distinction et ont tenté de l'exprimer. Il est également certain qu'un métalangage précis fait cruellement défaut à la langue française dans ce domaine, pénalisant ainsi l'appréhension des concepts par les enfants.

La dernière étape de cette réflexion a porté sur les différences sémantiques entre les deux valeurs du présent qui faisaient l'objet de leur observation. En travaillant sur deux phrases (demain je me déguise / demain je me déguiserai), les élèves ont émis plusieurs hypothèses : pour certains, il n'existe aucune différence sémantique; pour d'autres, le présent simple à valeur de futur exprime une proximité temporelle plus grande que le futur simple :

\begin{tabular}{|l|l|}
\hline 378. E.1: & c'est pas très loin \\
\hline 383. E.2: & le futur c'est euh c'est plus long / c'est plus loin c'est dans longtemps \\
\hline
\end{tabular}


Pour d'autres encore, il donne une impression de plus grande certitude :

\begin{tabular}{|l|l|}
\hline 367. E.1 : & si c'est peut-être on dit demain je me déguiserai \\
\hline 368. E.2 : & demain je me déguise / c'est plus sûr que demain je me déguiserai \\
\hline 412. I. : & quel effet ça vous fait si par exemple vos parents vous disent / cet été on va à la mer \\
\hline \hline 413. E.3: & c'est sûr \\
\hline
\end{tabular}

Difficile de dire si les sentiments de proximité / éloignement et certitude / incertitude des enfants sont recevables ou non. Ce sont leurs stratégies d'analyse métalinguistique qui nous intéressent principalement, et il semble qu'ils ont tenté de résoudre le problème posé, en faisant des efforts importants pour verbaliser leurs perceptions. Ils ont su dépasser le niveau intuitif et entrer dans des considérations métalinguistiques; les explications auxquelles ils ont abouti sont suffisamment claires pour qu'on les accepte en considérant qu'ils ont atteint, à leur niveau, l'objectif visé.

21 Ce travail mené avec un groupe de cours moyen montre qu'il est possible d'amener des enfants à réfléchir sur les subtilités du système temporel d'une langue, en l'occurrence le lien entre une forme verbale unique recouvrant plusieurs valeurs. Même sans métalangage académique, les élèves ont montré qu'ils pouvaient construire une réflexion, en procédant à des analyses logiques, mais aussi en utilisant leur intuition (sur l'acceptabilité d'un énoncé par exemple). Ceci n'est qu'un exemple des nombreux domaines linguistiques qu'il est possible d'explorer avec eux.

Dans le cadre d'une autre activité - qui précédait chronologiquement celle présentée cidessus -, diverses langues d'origine des enfants de cette classe ont été intégrées à la réflexion - l'italien, l'arabe, le turc et l'espagnol. Le travail portait alors sur l'analyse morphologique des formes verbales. Nous avons demandé aux enfants locuteurs d'italien de procéder à une analyse contrastive entre les systèmes morphologiques français et italien. Nous avons travaillé sur la base d'un exemple; en effet, un raisonnement général sur les modes d'expression de la temporalité en italien, étant impossible pour ces enfants, se serait probablement révélé peu fertile. Le but du travail était de mettre en évidence le principe de flexion en italien, en comparant un énoncé évoquant un phénomène présent et un énoncé évoquant le même phénomène, mais situé dans le futur. L'une des élèves s'est proposée d'exprimer l'exemple suivant :

- aujourd'hui, je joue avec mes amis

- demain, je jouerai avec mes amis

Elle a tout d'abord elle-même souligné que l'italien comportait, tout comme le français, des indicateurs de temps ayant pour rôle d'apporter des précisions sur l'événement prédiqué. Elle a ensuite produit, à l'oral, les phrases correspondantes en italien :

- oggi, gioco con i miei amici

- domani, giocerò con i miei amici

Le travail à l'oral a permis à toute la classe de s'intéresser au fonctionnement de cette langue, les enfants non-locuteurs d'italien ayant comme tâche de repérer les différences entre les deux énoncés, et de les caractériser en tentant de les répéter. Les 
réflexions des enfants ont été par exemple : il y a le premier mot qui change; il y a un petit bout en plus à la fin du mot $x$, etc.

La même réflexion a été menée sur l'arabe, le turc et l'espagnol. Il est à noter qu'une élève, locutrice d'espagnol, a eu beaucoup de mal à transposer les exemples dans sa langue d'origine, et n'a pas réussi à prendre conscience de la différence entre un énoncé présent et un énoncé futur. Ceci était peut-être dû à ses compétences plus rudimentaires en langue d'origine que les locuteurs d'italien, mais il est également probable que ses difficultés venaient de l'absence totale dans son vécu linguistique de réflexion d'ordre "méta " sur cette langue. Dans cette perspective, intégrer, dans un travail sur la temporalité, la L1 et les langues d'origine des apprenants apparaît ainsi comme une opération relativement bénéfique, ce qui peut se constater à quatre niveaux :

- pour les enfants locuteurs d'une langue d'origine, c'est l'occasion de prendre du recul par rapport à ce moyen de communication dont ils font un usage spontané en ayant une conscience très vague de son fonctionnement,

- c'est également l'occasion pour eux de prendre conscience de leur particularité au sein de la classe, et de manifester leur richesse linguistique ; le travail prend alors une dimension valorisante,

- les autres enfants, locuteurs d'autres langues d'origine ou non, peuvent ainsi découvrir des domaines linguistiques variés, inconnus jusqu'alors,

- le groupe dans son ensemble peut porter un regard différent sur sa L1 majoritaire, en la mettant en relation avec d'autres systèmes linguistiques.

\section{La représentation de l'accent}

Le deuxième exemple d'éveil à la conscience métalinguistique que nous aimerions présenter ici porte sur une notion fréquente, familière dans le langage courant, celle d'accent. Dans un contexte multilingue comme l'école où ce travail a été mis en place, c'est un phénomène langagier familier aux enfants, mais il semble qu'ils ne réfléchissent presque jamais à ses caractéristiques, ni à ce qu'il peut représenter socialement.

Dans le cadre d'un travail de réflexion sur les familles de langues, le problème s'est posé de définir la différence entre l'anglais et l'américain. Nous avons donc réfléchi sur les différents accents apparaissant au sein d'une même langue. Nous aimerions retracer ici le cheminement des enfants dans leur tentative de définition. Toujours grâce à un guidage par questionnement opéré conjointement par l'enseignante et l'enquêtrice, ils ont tenté de cerner les caractéristiques de ce phénomène en proposant diverses hypothèses. La dynamique du groupe a donc fonctionné autour des hypothèses émises par les uns, confirmées ou infirmées par les autres, utilisées comme base ultérieure de réflexion pour soumettre de nouvelles propositions. Six étapes ont été nécessaires aux enfants pour aboutir à une définition quelque peu exacte et précise. La première hypothèse, à la question " quelle est la différence entre l'anglais et l'américain », a été la suivante :

$$
\begin{aligned}
& \text { 105. } \\
& \text { C.: }
\end{aligned} \quad \text { alors quelle est la différence entre l'anglais et l'américain }
$$




\begin{tabular}{|c|c|}
\hline $\begin{array}{l}\text { 106. E. } \\
5:\end{array}$ & c'est que en anglais on met la langue et en américain on met pas la langue \\
\hline $\begin{array}{l}\text { 107. E. } \\
6:\end{array}$ & et ben non \\
\hline $\begin{array}{l}108 . \\
\text { C. : }\end{array}$ & $\begin{array}{l}\text { en anglais on met la langue et en américain on met pas la langue /[rires des autres enfants] c'est à } \\
\text { dire / tu parles sans ta langue }\end{array}$ \\
\hline $\begin{array}{l}\text { 109. E. } \\
\text { 3: }\end{array}$ & $\begin{array}{l}\text { non non mais on fait pas en anglais on fait euh / la langue elle touche les dents pour parler qu'en } \\
\text { américain non }\end{array}$ \\
\hline $\begin{array}{l}\text { 110. E. } \\
4:\end{array}$ & c'est l'accent qui est différent \\
\hline
\end{tabular}

Lorsqu'il parle de la «langue »-l'organe -, l'enfant détermine immédiatement que l'accent vient d'une différence purement articulatoire, donc physique, de l'accent. Il a toutefois du mal à préciser sa pensée, un autre élève intervient alors pour compléter la proposition de son camarade. Son explication est un peu vague, ou peut-être excessive, elle n'est donc pas retenue par les autres, et n'est pas confirmée par l'enquêtrice.

Les deux hypothèses suivantes sont liées à la voix - la hauteur dans le premier cas, le timbre dans le deuxième - :

\begin{tabular}{|l|l|}
\hline $\begin{array}{l}112 . \\
\text { C.: }\end{array}$ & qui est-ce qui peut me rappeler ce que c'est un accent \\
\hline $\begin{array}{l}113 . \\
\text { E.4: }\end{array}$ & c'est la différente façon de / de de parler / on peut parler grave ou aigu ça dépend \\
\hline $\begin{array}{l}114 . \\
\text { E.5: }\end{array}$ & on change un petit peu les mots \\
\hline $\begin{array}{l}115 . \\
\text { C.: }\end{array}$ & $\begin{array}{l}\text { non je ne suis pas d'ać qui a une voix très grave ce sera pas un accent différent / c'est pas ça un accent / ça c'est } \\
\text { une question de hauteur de la voix }\end{array}$ \\
\hline $\begin{array}{l}\text { (...) } \\
126 .\end{array}$ & oui alors les gens du midi / parlent / \\
\hline C.: & \\
\hline $\begin{array}{l}127 . \\
\text { E.3: }\end{array}$ & y zont un accent \\
\hline $\begin{array}{l}128 . \\
\text { E.1: }\end{array}$ & ouais ils ont l'accent marseillais \\
\hline
\end{tabular}




\begin{tabular}{|l|l|}
\hline $\begin{array}{l}\text { E.1: } \\
\text { E.1 }\end{array}$ & oui \\
\hline $\begin{array}{l}\text { E.2: } \\
\text { E1. }\end{array}$ & mais sauf qu'ils ont le timbre qui change \\
\hline [les enfants parlent en même temps - inaudible] \\
\hline $\begin{array}{l}\text { 132. } \\
\text { E.3: }\end{array}$ & le timbre on a tous un de différent \\
\hline $\begin{array}{l}\text { 133. } \\
\text { C.: }\end{array}$ & eh oui on a tous une voix différente / moi j'ai pas la même voix que toi \\
\hline
\end{tabular}

On peut observer ici que la recherche se poursuit, les enfants utilisant leurs connaissances empiriques de réalités phonétiques pour émettre des hypothèses. D'où l'apparition de termes tels que "grave », « aigu », ou «timbre ». Il est intéressant de noter un phénomène très fréquent dans ce type de séances, lié à la dynamique du groupe : l'échange n'est pas limité au schéma enseignant-élève, il existe aussi sous la forme élève-élève. Il est clair ici que les enfants travaillent collectivement, et prêtent attention à la réflexion de leurs pairs pour construire la leur. Le concept est progressivement découvert par le groupe, grâce à ce processus dynamique de construction d'hypothèses.

31 Dans les quatrième et cinquième hypothèses, les enfants manifestent leur conscience de la norme linguistique. En effet, un des élèves déclare qu'avoir un accent, c'est « compliquer » la langue de référence ( $y$ compliquent un petit peu) - il travaillait alors sur l'exemple de pain prononcé différemment par les marseillais -. Cette différence entre le français «standard» et l'accent du midi est vécue comme une déviation. De même, dans l'hypothèse suivante, un autre enfant déclare que les marseillais « rajoutent » des sons (ils rajoutent un [e] avant). Ils ressentent donc le phénomène d'accent comme un processus évolutif à partir d'une langue standard, non comme une différence existant per se. On peut observer dans ces exemples que la conscience métalinguistique s'affine, et que les enfants font preuve de beaucoup de ténacité pour aboutir à la définition exacte. Le principe de "déviation " n'est pas incohérent, car il est vrai qu'un accent n'est perçu que comme une différence par rapport à un autre type de prononciation. Cette particularité est essentielle sur le plan sociolinguistique, dans la mesure où un point de vue comparatif mène facilement à une échelle de valeur, qui est parfois à l'origine de dépréciation de variétés d'accents. Il est important que l'enfant prenne conscience que l'accent n'est perceptible que par comparaison, mais que cela n'implique pas nécessairement un jugement de valeur.

Un des élèves a finalement conclu que l'accent était une différence de «prononciation»:

\begin{tabular}{|l|l|}
\hline 150. E.5 : & $y$ changent la prononciation \\
\hline \hline 151. C. : & oui ils changent la prononciation du mot / bravo \\
\hline
\end{tabular}


152. E.6: c'est le même mot

33

\begin{abstract}
perçu où se situe la différence phonétique entre deux accents. Notre but n'étant pas de leur faire un cours de linguistique, nous avons retenu leur définition et nous avons tenté de l'illustrer de quelques exemples. Cette réflexion métalinguistique a ceci d'intéressant qu'elle a apporté beaucoup plus aux enfants que si l'enseignant avait d'emblée donné sa définition : elle leur a demandé une analyse individuelle du concept, la recherche d'exemples précis, un effort cognitif pour émettre des hypothèses, une attention particulière et un raisonnement pour analyser les hypothèses émises, une argumentation pour justifier leur rejet ou leur acceptation.

Cette activité réflexive menée avec les enfants sur les causes physiques de la différence entre les accents a débouché sur une discussion portant sur les représentations et l'attitude des enfants face à la différence langagière. Dans ce cadre, la réflexion métalinguistique a donc pour objectif, à partir de l'observation et l'étude de phénomènes langagiers, de développer la conscience méta-sociolinguistique des enfants à propos des problèmes de différence et de rejet, afin de les préparer à mieux vivre dans un milieu multilingue.
\end{abstract}

Reste à savoir bien sûr, malgré les félicitations de l'enseignante, et ses explications

\title{
3. L'ordre des mots
}

Le dernier exemple que nous aimerions présenter porte sur l'observation de l'ordre des items en aztèque. A partir de quelques exemples, les enfants ont pu procéder à une analyse de la place de l'adjectif - indiquant la taille ou l'âge - en aztèque, ainsi que la marque du pluriel. Ils ont réussi associer eux-mêmes certains noms et adjectifs, par déduction. Voici les exemples qui ont servi de base à la réflexion (Jones, 1984) :

\begin{tabular}{|l|l|l|}
\hline - ikalwewe & $=$ & grande maison \\
\hline - ikalsosol & $=$ & vieille maison \\
\hline - ikalcin & $=$ & petite maison \\
\hline & & \\
\hline - komitwewe & $=$ & grande casserole \\
\hline \hline - komitsosol & $=$ & vieille casserole \\
\hline - komitmeh & $=$ & des casseroles \\
\hline \hline & & \\
\hline - koyamecin & $=$ & petit cochon \\
\hline- koyamemeh & $=$ & des cochons \\
\hline
\end{tabular}



réflexion métalinguistique à deux niveaux: d'une part, la première étape de la séquence a mis en jeu des compétences cognitives essentielles dans l'émergence de la conscience. En effet, en analysant les points communs et les différences, les enfants ont su déduire les règles de fonctionnement de la langue dans cet exemple précis. D'autre part, la mise en regard de deux langues, dont l'une est utilisée comme norme de référence, leur a permis de toucher du doigt la variété des fonctionnements linguistiques, et placer la L1 sous un angle différent. de l'enfant. Ils nous montrent le rôle que peut jouer la confrontation avec des systèmes linguistiques étrangers dans le développement de la conscience. Dans cette perspective, 
il est particulièrement intéressant que cette confrontation s'exerce dans des cas de figure différenciés, que les systèmes en question soient totalement étrangers aux enfants ou qu'ils leur soient familiers. Ce travail montre ainsi qu'on a intérêt à construire cette conscience métalinguistique à l'intérieur d'un espace de plurilinguisme, et transversal à des situations individuelles différenciées. Les étapes de cette construction peuvent s'effectuer en l'absence d'un métalangage trop complexe en s'appuyant sur les instruments heuristiques dont dispose l'enfant à un moment de son apprentissage langagier.

\section{BIBLIOGRAPHIE}

BALBONI, P. (1993). «Language Awareness in the National Curriculum for Language Education in Italy ", in Language Awareness, vol. $2: 4: 190$.

CUMMINS, J. (1978). « Bilingualism and the development of metalinguistic awareness », in Journal of cross-cultural psychology, 9.

DABENE, L. (1991). « Enseignement précoce d'une langue ou éveil au langage ? » dans Enseignements/apprentissages précoces des langues (M. Garabédian dir.), Le Français dans le monde, Recherches et applications : 57-64.

DABENE, L. (1992). « Le développement de la conscience métalinguistique : un objectif commun pour l'enseignement de la langue maternelle et des langues étrangères », in Repères, 6 : 13-21.

DABENE, L. (1994). Repères sociolinguistiques pour la didactique des langues, Paris, Hachette.

GOMBERT, J-E. (1990). Le développement métalinguistique. Paris, Presses Universitaires de France.

HAKES, D.T., EVANS, J.S. et TUNMER, W.E. (1980). The development of metalinguistic abilities in children, Berlin, Springer-Verlag.

HAMERS, J.F. et BLANC, M. (1983). Bilingualité et bilinguisme, Bruxelles, Mardaga.

HAWKINS, E. (1984). Awareness of Language an introduction, Cambridge University Press.

JAMES, C. et GARRETT, P. (eds), (1991). Language Awareness in the Classroom.. Londres, Longman. JONES, B. (1984) How Language Works, Awareness of Language, Cambridge University Press : 8. NAGY, C. (1993). «Organisation de l'interaction et mise en œuvre de la conceptualisation dans une classe d'éveil au langage ", in Les pratiques de classe en langue étrangère, Actes du $3^{\circ}$ colloque international ACEDLE, Ecole Nationale Supérieure de St Cloud : 117-127.

PRATT, C. et GRIEVE, R. (1984) « The development of metalinguistic awareness : an introduction ", in Tunmer, W.E., Pratt, C. \& Herriman, M.L. (Eds), Metalinguistic Awareness in Children, Berlin, Springer Verlag : 6 . 


\section{RÉSUMÉS}

On se propose, dans la présente contribution, de mettre en évidence les traces de l'émergence, chez l'enfant d'âge scolaire, de la conscience métalinguistique, à travers diverses activités telles que le contact avec des systèmes linguistiques diversifiés ou l'observation concrète de certains phénomènes langagiers.

Seront ainsi analysés des exemples d'interactions menées dans la classe à propos de notions telles que la temporalité, l'accent ou l'ordre des mots. On s'attachera à mettre en évidence ce qui manifeste, chez les sujets étudiés, la prise de conscience progressive de l'univers du langage dans certaines de ses caractéristiques jugées pertinentes pour des apprentissages futurs.

In this article, we retrace the emergence of metalinguistic awareness in school children through the examination of language contact and other relevant phenomena. To this end, we analyse instances of classroom interaction and attempt to identify the developing linguistic awareness of these subjects for temporality. Word order and accent.

\section{AUTEURS}

\section{LOUISE DABÈNE}

Centre de Didactique des Langues

Université Stendhal-Grenoble III

\section{CHRISTÈLE INGELMANN}

Centre de Didactique des Langues

Université Stendhal-Grenoble III 